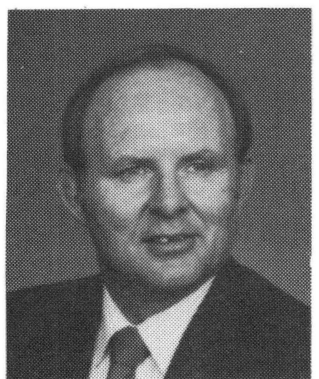

\section{Editorial}

Jack D. Gaskill, Editor

When You Agree to Be a Referee

The stature of a technical journal is determined by the quality of the papers it publishes, which in turn depends upon both the quality of the papers submitted and the quality of the reviews those papers receive. In this editorial I offer a number of suggestions that I hope will assist referees of Optical Engineering papers to provide objective, thorough, and helpful reviews that I can then use in reaching a decision to publish or reject and that authors can use to revise and improve their papers.

If you are asked to serve as a referee, the first thing you can do to help me is to promptly let me know whether or not you will honor my request. If the answer is no for any reason-if you believe that you are unqualified to review that paper, if you feel that you have a conflict of interest and may be unable to review it objectively, or if you are simply too busy to review it in a timely fashion-please return the entire package to me immediately in the large prestamped envelope provided. It will also help me if you can suggest the name and address of at least one other qualified referee.

If, on the other hand, you decide that you will be able to provide an objective and timely review, please so indicate on the prestamped card that is provided and return it to me immediately.

Next, evaluate the paper by considering the following aspects:

(1) Do the title and abstract accurately reflect the contents of the paper?

(2) Is the paper well written and easy to read? Is it well organized?

(3) Does it have merit? Does it make a contribution to the field?
(4) Are pertinent prior publications on the subject satisfactorily cited?

(5) Does it contain any serious errors? Are the assumptions reasonable? Are the approximations valid?

(6) Are the illustrations necessary? Do they help the reader?

(7) Are the conclusions correct? Are there flaws in the reasoning leading to these conclusions?

(8) Do you recommend publication in Optical Engineering? If not, can you recommend another publication for which the paper might be suitable?

When you have completed your assessment of the paper, it would be helpful if you would prepare an anonymous report that I can send directly to the author(s). This report should address the items listed above, as well as any others that you deem important, and should be written in as positive and constructive a manner as possible. If you have criticisms to make, try to word them in such a way that you would find them to be both useful and inoffensive if you were the author. As you probably already know, it requires skill to offer criticism without being offensive. If you have additional comments that you do not want the author to see, please include them in your cover letter to me and they will not be sent on to the author.

I have a suggestion that will make the review process a little less painful and time consuming: resist the temptation to be a copy editor. I realize that this may be difficult for some of you (it is for me), but I'm interested in your technical expertise and judgment rather than your editing skills. Between Martha Stockton and the staff in Bellingham, we already have a team that could win a gold medal in the editorial Olympics. It can certainly be helpful for you to comment on the style, grammar, etc., of the paper, but I recommend that you leave the "blue pencil" work to the copy editors.

I would also like to point out that, while I am hoping for a critical review, you shouldn't feel compelled to search and search for an error until you find one or to make a big fuss about a minor imperfection. After all, a great many of the papers we receive are very good just the way they, are submitted.

Finally, I would like to once again thank all of the referees who have provided prompt, professional reviews in the past, and to thank all of those referees-before the fact-who will do so in the future.

\title{
Special Issue Call for Papers
}

\section{May 1989}

\section{Industrial Applications of Optical Signal Processing III}

\section{Guest Editor}

Bahram Javidi

University of Connecticut

Department of Electrical Engineering

Storrs, CT $06268 \quad$ 203/486-4816

The May 1989 special issue of Optical Engineering will be devoted to the continuation of our series of special issues on Industrial Applications of Optical Signal Processing. This special issue will be devoted to architectures and systems that can be used in industrial applications that require advanced optical signal processing techniques. The issue will cover the following areas: optical information processing, including linear, nonlinear, and space variant operations and transforms; pattern recognition, correlation, hybrid optical/digital systems, multiclass distortion invariant object identification, and related applications of optical architectures for associative memories; automatic object inspection, feature extraction, and classification; applications for holography, including computer-generated holography; and acousto-optic signal processing systems.

Papers will also be included on applications of optical techniques to systems that require adaptive massively parallel, fault tolerant capabilities using the implementation of associative memory and neural networks. These include adaptive pattern recognition models, competitive learning, image processing models, specialized neural network models, computational analysis, and their optoelectronic implementation.

Authors are encouraged to submit manuscripts on any of the above topics for inclusion in the special issue. Manuscripts submitted for consideration should be sent to the Guest Editor before October 15, 1988.

Additional calls for papers on pp. 739 and 748; complete editorial schedule on page 735 . 